\title{
Un acercamiento a la historia de la documentación científica en Cataluña. La labor de María Serrallach Juliá (1905-1992) en el Seminario de Química de la Universidad de Barcelona (1937-1984)
}

\author{
Guillermo Olagüe de Ros (*) \\ ${ }^{*}$ ) Departamento de Historia de la Ciencia, Universidad de Granada, Granada. \\ golague@ugr.es
}

Dynamis

[0211-9536] 2014; 34 (1): 147-168

http://dx.doi.org/10.4321/S0211-95362014000100008
Fecha de recepción: 25 de septiembre de 2012

Fecha de aceptación: 19 de febrero de 2013

SUMARIO: 1.-María Serrallach Juliá (1905-1992), una bibliotecaria especializada. 2.—Las publicaciones 3.—La actividad docente. 4.—El Seminario de Química (1937-1984). 5.—Colofón.

RESUMEN: En este trabajo se ofrece un estudio sobre María Serrallach Juliá (1905-1992) una de las primeras bibliotecarias especializadas de nuestro país que, entre 1937 y 1975, dirigió el Seminario de Química de la Universidad de Barcelona (SQUB), una biblioteca resultante de la fusión de las de las Facultades de Farmacia y Químicas de la Universidad de Barcelona. Serrallach convirtió el SQUB en un moderno centro de documentación científica, pues ofertó servicios que eran prácticamente inexistentes en el resto de España. En el artículo se estudian también las publicaciones y la actividad docente de Serrallach y se analiza la evolución del SQUB hasta 1984, en que se transformó en la Biblioteca de las Facultades de Física y Química de dicha Universidad catalana.

PALABRAS CLAVE: historia de la documentación científica, España, siglo XX, Cataluña, Seminario de Química, Universidad de Barcelona, María Serrallach Juliá.

KEY WORDS: history of scientific information, Spain, 20th Century, Catalonia, Seminar of Chemistry, University of Barcelona, María Serrallach Juliá.

A partir de la década de los 60 de la pasada centuria, conforme a la tónica generalizada en otros países, se produce en España un espectacular crecimiento de servicios o centros de documentación científica. Con el 
paso de los años algunos de ellos adquirieron prestigio y se convirtieron en referentes en sus áreas de conocimiento, mientras que otros apenas tuvieron continuidad. La culminación de este proceso se dio en julio de 1975 cuando, como consecuencia de directrices emanadas por organismos internacionales, se constituyó el CENIDOC, Centro Nacional de Información y Documentación Científica, a partir de tres de esos servicios ya existentes: el Instituto de Ciencia y Tecnología (ICYT), el Instituto de Ciencias Sociales y Humanidades (ISOC), del Consejo Superior de Investigaciones Científicas (CSIC), con sede en Madrid, y el Instituto de Documentación e Información en Biomedicina, ubicado en la Facultad de Medicina de Valencia. Con la creación del CENIDOC teóricamente quedaban cubiertas las necesidades informativas de los estudiosos en todas las ramas científicas $\mathrm{y}$ en las humanidades.

Aunque está por hacer un estudio en profundidad sobre los orígenes y evolución posterior del CENIDOC, el comienzo y desarrollo de la documentación científica en Cataluña es si cabe menos conocido. Este trabajo pretende, precisamente, ofrecer una primera aproximación al progreso documental en Cataluña, concretamente a la labor en química llevada a cabo por María Serrallach Juliá (1905-1992), una bibliotecaria formada en la Escola Superior de Bibliotecàries de Barcelona, que dirigió durante casi cuarenta años el Seminario de Química de la Universidad de Barcelona (SQUB), una biblioteca creada en 1937 y que a partir de 1942 - por primera vez en España- comenzó a prestar servicios documentales a sus usuarios.

Serrallach es una de las primeras special librarians de nuestro país; es decir, una bibliotecaria experta en la recuperación y difusión de los fondos contenidos en una biblioteca especializada, en su caso científico-técnica. La contraposición entre el bibliotecario conservador de fondos bibliográficos y el nuevo documentalista perito en el tratamiento informativo, la ha ejemplificado muy certeramente Farkas-Conn al comparar históricamente los programas primo seculares de Melvil Dewey (1851-1931), más preocupado por la catalogación, y de Paul Otlet (1868-1944) y Henri Lafontaine (1854-1943), con una visión más documental ${ }^{1}$.

1. Farkas-Conn, Irene. From Documentation to Information Science. Westport: Greenwood Press; 1990, p. 4. Está por hacer la historia de los diferentes modelos de bibliotecarios españoles a lo largo del siglo XX y el papel adoptado por algunos de ellos en la difusión de la documentación como disciplina en nuestro país. En 1961 la revista del Cuerpo de Archiveros y Bibliotecarios inauguraba una sección, «Documentación», que se abrió con un clarificador 
La evolución de la documentación científica en Cataluña presenta cierto paralelismo con la que tuvo lugar en Madrid, el otro gran polo documental español. Como elementos comunes señalaré, entre otros, la larga tradición desde principios del siglo XX en la confección de repertorios de bibliografía corriente, especialmente en biomedicina, algunos de ellos ligados a las grandes corrientes documentales de la época; los intentos a partir de los años 70 de coordinar servicios documentales, bien con carácter global o en áreas concretas; la incorporación de revolucionarios sistemas de recuperación de la información, como la teledocumentación, a partir de 1973 en Madrid y desde el año siguiente en Cataluña; o la preocupación compartida por Madrid y Barcelona acerca de las patentes industriales, que se tradujo en la creación de oficinas especializadas en el registro y explotación de las mismas, Madrid más precozmente y Cataluña en fechas posteriores.

Pero Cataluña contó con algunas singularidades que merecen ser consideradas. Por un lado, la presencia desde 1915 de una docencia reglada en biblioteconomía en la ya citada Escola Superior de Bibliotècaries de Barcelona, una experiencia muy novedosa y de la que surgieron algunas de las responsables de los servicios documentales científicos tras la Guerra Civil, como María Serrallach, o defensoras del modelo de bibliotecaria especializada, como Mercedes Rosell Rosal ${ }^{2}$. La Escola tuvo tempranamente gran interés por la enseñanza de la información y documentación científicas antes incluso que en el resto de España. Esta tradición ha proseguido hasta la fecha en el mundo universitario catalán, mientras que en el resto del Estado ha tenido una evolución desigual. La publicación desde la década de los ochenta de varios manuales de documentación química es consecuencia de ese interés por la disciplina.

La segunda característica es el especial desarrollo documental en algunas ramas científicas, en buena medida ligado a su crecimiento industrial. En el caso de la química, por ejemplo, Madrid tuvo también importantes referentes, pero en otros, como la ingeniería, Cataluña fue pionera. Incluso en química, Barcelona contó con servicios muy tempranamente, antes

artículo de Javier Lasso de la Vega, en el que defendía la documentación como materia propia de los bibliotecarios, y la necesidad de un buen conocimiento de idiomas vivos y de las modernas técnicas bibliotecológicas (La Documentación. Un sólido futuro para la profesión bibliotecaria. Boletín de la Dirección General de Archivos y Bibliotecas. 1961; 10 (59): 41-44).

2. Rosell, Mercedes. La documentación y las bibliotecas especializadas. Biblioteconomía. 1963; 20 (57-58): 26-31. En la misma línea está otro artículo de María Serrallach: Centros de Documentación. Biblioteconomía. 1963; 20 (57-58):19-25. 
incluso que Madrid, surgidos en el mundo universitario y que buscaron la colaboración financiera de las empresas de su entorno para su sostenimiento, como el SQUB o el Instituto Químico de Sarriá (IQS), un centro docente privado fundado por los jesuitas en $1916^{3}$. Conviene recordar, además, que en 1955 se editó uno de los primeros catálogos contemporáneos de libros de química, que apareció como un número extraordinario de la revista Afinidad, editada por la Asociación de Químicos e Ingenieros del IQS, en el que se recogían 1.200 referencias ${ }^{4}$.

Este importante desarrollo documental catalán también se manifestó con la celebración en Barcelona de, probablemente, los primeros congresos en nuestro país sobre esta especialidad ${ }^{5}$. En noviembre de 1972, y organizado por el IQS, tenía lugar un «Coloquio Hispano-Francés sobre Información y Documentación Científica y Técnica», que conoció una segunda edición en Toulouse en febrero de 1977. En marzo de 1973 se desarrollaban las «Primeras Jornadas sobre Documentación y Bibliografía Química», organizadas por la Asociación Nacional de Químicos de España, en las que intervinieron entre otros expertos María Serrallach y José Ramón Pérez Álvarez-Ossorio, director del Centro de Documentación e Información del CSIC, antecesor inmediato del CENIDOC 6 . Unos meses después, en diciembre, el Centro de

3. López García, Santiago M.; Puig Raposo, Núria. Ciència i industria a Catalunya. L'impacte de I'Institut Químic de Sarrià a la indústria catalana del segle XX. In: Navarro Brotóns, Víctor; Salavert Fabiani, Vicent L.; Corell Domènech, Mavi et al., coords. Actes de les II Trobades d'Història de la Ciència i de la Tècnica. Barcelona: Institut d'Estudis Catalans; 1993, p. 207-213.

4. Catálogo del Libro Español de Química (1920-1955). Afinidad. 1955; Número extraordinario (fuera de serie).

5. En la década de los 60 tuvieron lugar en el CSIC de Madrid dos actividades importantes no congresuales en el ámbito de la documentación científica. Por un lado, el ciclo de conferencias impartidas en el Patronato Juan de la Cierva por Erich Pietsch en 1960 y que se publicaron como monografía ese año (Información y Documentación: Naturaleza y posibilidades. Madrid: CSIC). Por otro, el simposio celebrado en marzo de 1965 sobre nuevos procedimientos documentales, en el que Eugene Garfield y Leo J. Stevens — del Institute of Scientific Information-, presentaron el Science Citation Index y el Automatic Subject Citation Alert. Simposio sobre modernos procedimientos. Revista de Ciencia Aplicada. 1965; 19 (1): 176.

6. Coloquio Hispano-Francés sobre la Información y la Documentación Científico y Técnica, base de la innovación tecnológica. Afinidad. 1973; 30 (307): 473-559, que recoge las 14 ponencias presentadas, nueve de españoles y cinco de especialistas franceses. Serrallach no participó. Sobre las Jornadas únicamente he podido localizar las aportaciones de Serrallach y Pérez Álvarez-Ossorio, que aparecieron de forma independiente. Serrallach, María. La Biblioteca Química. Barcelona: Imprenta de la Universidad; 1973. Un año antes María había editado prácticamente este texto en la revista Biblioteconomía [29 (75-76): 17-30]. Pérez Álvarez- 
Documentación en Ingeniería creado ese mismo año por el Colegio Oficial de Ingenieros Industriales de Cataluña, organizaba unas «ornadas sobre información y documentación al servicio del ingeniero y de la empresa» ${ }^{7}$.

Sin embargo, ninguno de estos centros documentales de Cataluña fue considerado a la hora de la creación del CENIDOC ni en las posteriores fundaciones de rango nacional.

\section{María Serrallach Juliá (1905-1992), una bibliotecaria especializada}

El estallido de la Guerra Civil no interrumpió la vida académica universitaria catalana. En 1937 las Facultades de Química y Farmacia sumaron sus fondos bibliográficos en una única biblioteca, el SQUB, que como tal funcionó hasta 1984. Durante sus casi cincuenta años de actividad la responsabilidad académica del mismo recayó alternativamente en profesores de Farmacia o de Químicas ${ }^{8}$, pero su funcionamiento dependió desde el principio de María Serrallach Juliá, que hizo del Seminario la mejor biblioteca físico-química del Estado.

María Serrallach nació en una familia de larga tradición médica que continúa en la actualidad. Narciso Serrallach Maurí (1875-1951), padre de María, fue uno de los fundadores de la urología en nuestro país ${ }^{9}$. Dos de los hermanos de María alcanzaron bastante notoriedad. José Antonio (+1989), químico, tras una estancia en el Instituto Tecnológico de Massachusetts fundó en 1934 el laboratorio farmacéutico LAINCO, desde el que se comercializaron productos tan exitosos como el laxante Emuliquen o el antiséptico dérmico Mercromina. Francisco (1899-1986) siguió la profesión paterna y alcanzó también gran renombre en esa especialidad médica.

Ossorio, José Ramón. Panorámica mundial de los servicios de Información y Documentación en Química. Ciencia y Técnica en el Mundo. 1973; 25 (419): 565-586.

7. Queralt Teixidó, Rafael; Tomás Morer, Xavier. La formación de los usuarios de información científica y técnica. Revista Española de Documentación Científica. 1979; 1 (5): 475-476.

8. El primer profesor responsable fue Enrique Soler y Batlle (1877-1951), de la Facultad de Farmacia, al que le sucedieron los profesores de Químicas José Pascual Vila (1895-1979) y José Costa López (n. 1936).

9. Serrallach Milá, Narcis; Serrallach Orejas, Francesc; Serrallach Orejas, Marc. Hojas Urológicas del Dr. Narciso Serrallach Maurí, escritas hace casi cien años. Archivos Españoles de Urología. 2007; 60 (8): 958-972. 
María estudió en la Escuela Alemana de Barcelona, en la calle Moià. De esa época, en torno a 1915-16, recuerda María su relación con otra alumna de la Escuela, Margarita Braunstein, en realidad Zinaida Bronstein (1901-1933), hija de León Trotsky, con la que mantuvo una estrecha amistad hasta que ésta marchó a América ${ }^{10}$. En 1930 María obtuvo el título de Tenedor de Libros por la Academia Práctica de Barcelona ${ }^{11}$. Tres años después, tras presentar su trabajo de fin de estudios sobre las bibliotecas en la extinta Unión Soviética, se diplomaba por la Escola de Bibliotecàries de Barcelona ${ }^{12}$. No he podido verificar que entre 1933 y 1936 trabajara en la Biblioteca del Tir Nacional ${ }^{13}$. Al siguiente año, como ya señalé, fue requerida por la Facultad de Farmacia para ponerse al frente del recién creado SQUB por su formación bibliotecaria y por su amplio conocimiento de idiomas ${ }^{14}$. En esos duros años de la Guerra Civil María se dedicó también a proteger algunos fondos bibliográficos catalanes, por ejemplo las bibliotecas del Convento de los Capuchinos de Sarriá, la del Seminario Menor de la Conrería (Tiana), que fue trasladada a Badalona, ambas a instancias de Jordi Rubió i Balaguer (1887-1982), y la del IQS, que María acogió en el SQUB y que reintegró a los Jesuitas en $1940^{15}$.

10. Serrallach, María. Trotsky, en Barcelona. Evocación de una amistad escolar. La Vanguardia Española. 15 Ene 1970: 47.

11. La Vanguardia Española. 2 Ago 1930: 8.

12. Planet, Anna. En mèmoria de la María Serrallach. Item. 1992; 11: 156-158.

13. Retrats de dones. Xarxa europea de dones periodistas. Bibliotecàries pioneres. Maria Serrallach Julià (Barcelona, 1905-1992) [citado 6 Sep 2012]. Disponible en: http://www.mujeresperiodistas. net/menuRetratsDones.htm.

14. Ya en 1935 obtuvo una beca de dos meses para perfeccionar el inglés en la Universidad de Londres (Destino. 1965; n. 1473: 11). Al poco de obtener la plaza de bibliotecaria superó las pruebas de traductora jurada de inglés y alemán (Planet, n. 12, 156). Como señalaría María en una entrevista concedida a su regreso de los Estados Unidos, conocía «inglés, francés, alemán, español, italiano y algo entiendo de ruso». Del Arco. Mano a Mano. María Serrallach. La Vanguardia Española. 29 Dic 1957: 27.

15. Gudayol, Anna. El salvament de les biblioteques catalanes durant la Guerra Civil: Les «Notes» de Jordi Rubió. Estudis Romanics. 2007; 29: 213-224; Serrallach, María. Mis vivencias en la Biblioteca del Seminario de Química de la Universidad de Barcelona [1975] 17 f. Localizado en: Biblioteca Universitaria, Barcelona. [citado 8 Feb 2012]. Disponible en: www.bib.ub.edu/ fileadmin/bibs/fisica_quimica/discurs.pdf. 


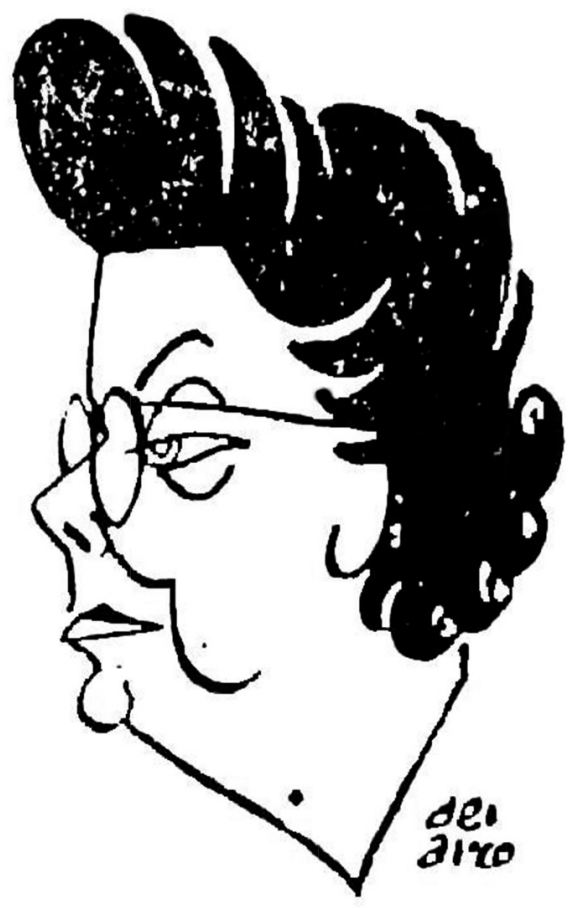

Figura 1.-María Serrallach Juliá, vista por Manuel del Arco. Fuente: Del Arco. Mano a Mano. María Serrallach. La Vanguardia Española. 29 diciembre 1957: 27.

Aunque María tenía una buena formación en sistemas de catalogación, fundamentalmente en la Clasificación Decimal Universal (CDU), pues el conocimiento de la misma era materia docente en la Escola, algunos de los promotores del SQUB, como José Pascual Vila, le hicieron ver la necesidad de recurrir a otros sistemas clasificatorios, pues entendían que la CDU no era adecuada para ordenar fondos científicos, y menos los de una biblioteca como la del SQUB, que pretendía ser «de alta investigación», es decir, sin monografías pero con «obras de información y referencia y con colecciones de revistas» ${ }^{16}$. Pero a pesar de esta recomendación,

16. Serrallach, n. 15, f. 2-3. Sobre la importancia de la CDU en la revista Biblioteconomía: Vall Casas, Aurora. La revista Biblioteconomía (1944-1976) o com I'anàlisi del contingut i els llenguatges 
María recurrió a la CDU para la ordenación de las materias y revistas del SQUB, tal como se recoge en las memorias académicas de la Universidad de Barcelona de los años 60.

Para garantizar su continuidad en el SQUB, las Facultades de Farmacia y Química le sugirieron que se presentara a las oposiciones del Cuerpo Auxiliar de Archivos y Bibliotecas, pues dado que no era Licenciada en Letras no podía aspirar al Cuerpo Facultativo. Conseguida la plaza, ingresó en el escalafón el 16 de mayo de 1941 y pidió como destino el SQUB, en el que trabajó hasta su jubilación oficial en 1975, aunque siguió colaborando con esta biblioteca durante un quinquenio más. Dos años antes de que ésta se produjera, en 1973, la Dirección General de Archivos y Bibliotecas denegó su nombramiento como Jefa de Servicios por no ser licenciada. La propuesta había sido apoyada por notorias personalidades universitarias y científicas, por diversos organismos y por Rosalía Guilleumas i Brosa (1924-2007), superiora jerárquica de María y máxima autoridad en el sistema bibliotecario catalán ${ }^{17}$.

A partir de 1942, según narra María Serrallach, el SQUB comenzó a transformarse en un servicio de documentación científica, que ofrecía copia de los trabajos que interesaban a los usuarios de la biblioteca, o bien eran solicitados a otros centros nacionales o del extranjero. Para las traducciones era la propia María la que se encargaba de hacerlas, fuera del horario laboral, y para las búsquedas bibliográficas contó con la colaboración de un licenciado. Además, muy tempranamente, el SQUB dispuso de unos rudimentarios aparatos para fotocopiar y microfilmar los artículos que precisaban los lectores, en cuya construcción participó uno de sus hermanos, quizás los primeros de una biblioteca científica en España ${ }^{18}$. Desligada ya del Seminario, se consiguió en 1981 la instalación de una terminal para consultas teledocumentales de bases de datos bibliográficas ${ }^{19}$.

documentals interessaven als professionals de l'època. BiD [publicación seriada en internet]. 2004; n. 13. [citado 15 Jul 2012]. Disponible en: http://www.ub.edu/bid/13vall.htm

17. Serrallach, n. 15, f. 3 .

18. Serrallach, n. 15, f. 8-9.

19. Alcolea, Santiago; Lamarca, Dolors; Llopart, Pilar et al. La Biblioteca de la Universitat de BarceIona. Barcelona: Universitat; 1994, p. 71. 


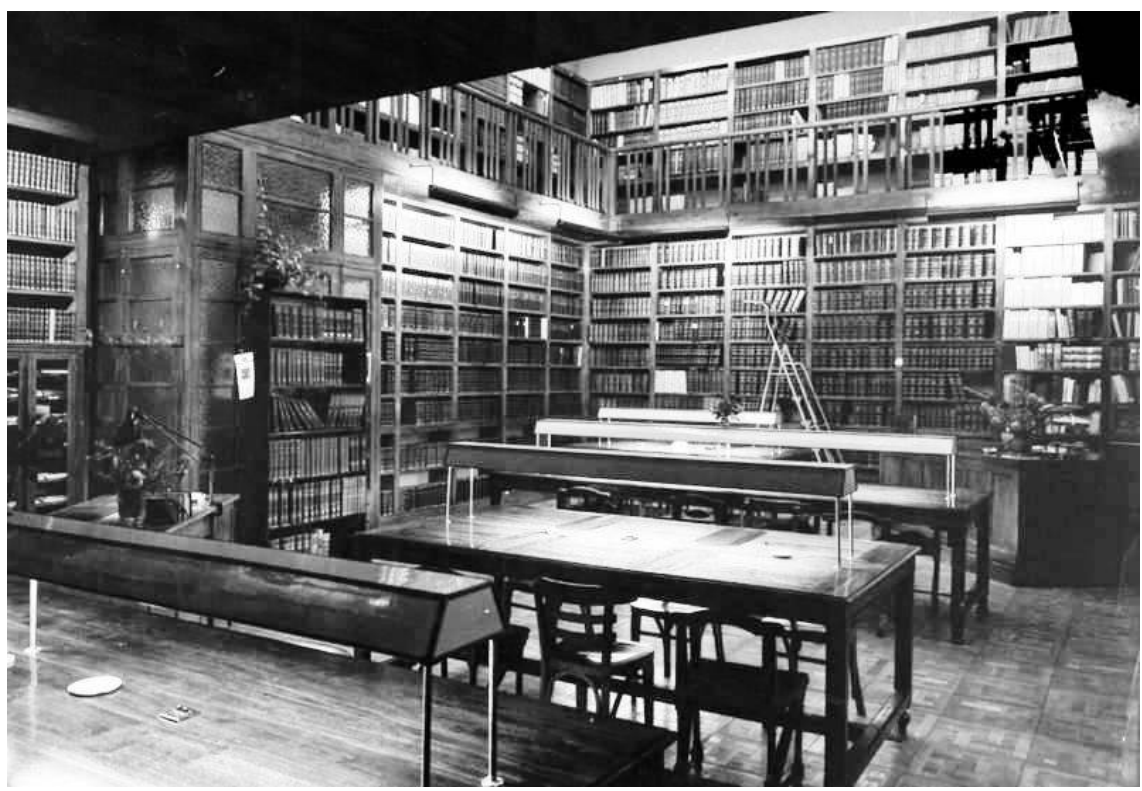

Figura 2.-La Biblioteca del Seminario de Química de la Universidad de Barcelona, en torno a los años 60. Fuente: http://www.bib.ub.edu/biblioteques/fisica-quimica/mostres-bibliografiques/ centenari-chemical-abstracts/100-anys-a-la-biblioteca/?type=98.

En 1956, María Serrallach obtuvo una beca del Jointly-Sponsored Program for Foreign Librarians, un proyecto promovido ese año por el Departamento de Estado, la Library of Congress y por las dos asociaciones bibliotecarias mayoritarias de los Estados Unidos, la Special Libraries y la American Library, al objeto de atraer a las bibliotecas norteamericanas a profesionales de otros países ${ }^{20}$. María consiguió la subvención para trabajar en la biblioteca de Ciencias de la Universidad Católica de Notre Dame, en Indiana, un importante centro con más de 45.000 volúmenes ${ }^{21}$. Esta prolongada estancia, desde enero de 1957, le permitió conocer, además, casi cuarenta bibliotecas

20. El programa estuvo operativo entre 1956 y 1971. De las 52 becarias de la primera convocatoria, María fue la única española, junto con otras siete bibliotecarias procedentes de India, Israel, Filipinas, Vietnam, Suecia, Brasil y Perú. Sobre este programa: Allaway, Jean D. Post mortem: The Jointly Sponsored Program for foreign librarians. Special Libraries. 1972; 63 (7): 313-317.

21. Bledsoe, E. A. Spanish special librarian arrives under Jointly-Sponsored Program. Special Libraries. 1957; 48 (2): 74. 
y centros documentales norteamericanos, como la Library of Congress, la Public Library de Nueva York, la John Crear de la Universidad de Chicago y varias californianas, entre otras. Seis meses después de su llegada a Indiana, Victor A. Schaefler, director de la Biblioteca Universitaria, se pronunciaba muy favorablemente sobre la presencia de María en su Universidad ${ }^{22}$. De las visitas que realizó Serrallach quedó muy impresionada con las oficinas del Chemical Abstracts, ubicadas en un edificio propio en el campus de la Ohio State University, en Columbus (Ohio). A su regreso a España María publicó el primer artículo editado en nuestro país sobre este repertorio, una completa nota que apareció en la revista madrileña Ion, editada por el Sindicato Nacional de Industrias Químicas ${ }^{23}$.

La estancia americana fue para María Serrallach muy determinante desde el punto de vista formativo y profesional, pues le permitió conocer los más modernos sistemas bibliotecológicos de los Estados Unidos y acomodarlos a la biblioteca del SQUB. Un detallado informe sobre la labor realizada y su favorable visión del mundo bibliotecario de Norteamérica lo ofreció en 1959 en el Boletín de la Dirección General de Archivos y Bibliotecas, revista en la que además publicó varios artículos sobre la cuestión que más adelante comentaré ${ }^{24}$. Además consiguió que la United States Book Exchange Inc., un organismo privado sin ánimo de lucro con sede en la Library of Congress en Washington, donara gratuitamente al SQUB un importante lote de revistas científicas, básicamente de química, por un valor superior al medio millón de pesetas y con un peso aproximado de tonelada y media. Con tal motivo se organizó una exposición en el propio Seminario, a la que asistieron las máximas autoridades académicas de la Universidad, así como el cónsul de los Estados Unidos en Barcelona, el señor Carney ${ }^{25}$. También

22. «We are quite happy with Miss Serrallach and agree (...) that the matching of candidates with the library has certainly turned out very well for us at Notre Dame». Special Libraries. 1957; 48 (6): 272.

23. Serrallach, María. El «Chemical Abstracts». Su historia, instalación y actividades. Ion. 1957; 17 (193): 468-470.

24. Serrallach, María. Actividades relacionadas con la beca obtenida por María Serrallach para los Estados Unidos. Boletín de la Dirección General de Archivos y Bibliotecas. 1959; 8 (50): 32.

25. Exposición de un donativo de revistas técnicas al Seminario de Química. La Vanguardia Española. 1 Jul 1959: 20. El Boletín de la Dirección General de Archivos y Bibliotecas ofreció una noticia con dos fotografías de la exposición, la relación nominal de revistas recibidas hasta la fecha y años de cobertura. Exposición del donativo de revistas hecho por el United States Boock (sic) Exchange Inc. de Washington, a la Biblioteca del Seminario de Química de la Universidad de Barcelona. Boletín de la Dirección General de Archivos y Bibliotecas. 1959; 8 (51): 27-28. 
fueron abundantes las conferencias que impartió en distintos foros sobre los profesionales y el mundo de las bibliotecas de los Estados Unidos ${ }^{26}$.

A mediados de los sesenta la labor de María en el SQUB empezó a ser reconocida en diversos medios. En la apertura del curso académico 1965-1966 se le hizo entrega de la Encomienda con Placa de la Orden de Alfonso X el Sabio por su trabajo al frente de la Biblioteca de Química de la Universidad ${ }^{27}$. Miguel Masriera y Rubio (1901-1981), profesor de QuímicaFísica en la Universidad de Barcelona, en dos artículos publicados con un intervalo de diez años en La Vanguardia sobre bibliografía científica (1966 y 1976), ensalzaba la importante labor que María Serrallach venía realizando desde hacía varios años en el SQUB:

«Tenemos un magnífico y encomiable... Seminario de Química de nuestra Universidad. Se inició... durante nuestra guerra civil. La señorita María Serrallach... con su trabajo ímprobo puso la biblioteca al día y desde entonces, con dedicación ejemplar y un celo casi atrabiliario y que por desgracia no es corriente ver en defensa de intereses ajenos, ha conseguido... con abnegadas colaboradoras, tener ahora actualizada la biblioteca del Seminario» ${ }^{28}$.

El SQUB se convirtió, además, en un centro de atracción para los bibliotecarios que querían conocer una biblioteca especializada moderna. Por ejemplo, el 13 de junio de 1966 los participantes en el XII Curso de

26. Una nómina de dichas conferencias en: Serrallach, nota 24.

27. Boletín Oficial del Estado. 4 agosto 1965; (185): 10.983; De la apertura del curso en la Universidad. La Vanguardia Española. 6 Oct 1965: 21; En la Universidad de Barcelona. ABC (Madrid). 5 Oct 1965: 67.

28. Masriera, Miguel. ¿A dónde va la ciencia? Bibliografía Babélica. La Vanguardia Española. 1 febrero 1966: 9, y El Futuro de la Ciencia (1). Bibliografía Neoplásica. La Vanguardia Española. 27 enero 1976: 15. El primero de estos dos artículos lo reprodujo, además, el Boletín de la Dirección General de Archivos y Bibliotecas. 1966; 15 (87): 27-28. Últimamente Miguel Masriera ha merecido la atención de Agustí Nieto-Galán, que en dos trabajos ha subrayado la importancia de Masriera como periodista científico. Químico de formación, Masriera inició en los años 20 de la pasada centuria una labor divulgadora sobre temas científicos en la prensa. Tas la conclusión de la Guerra Civil se exilió a Francia y cuando decidió regresar a España le fueron cerradas las puertas de la Universidad y del Consejo Superior de Investigaciones Científicas. Nieto-Galán, Agustí. Miguel Masriera (1901-1981) y la divulgación de la física nuclear en la España de Franco. In: Herran, Néstor; Roqué, Xavier, eds. La física en la dictadura. Físicos, cultura y poder en España 1939-1975. Bellaterra: Servei de Publicacions, Universitat Autònoma de Barcelona; 2012, p. 277-293; Nieto-Galán, Agustí. From papers to newspapers: Miguel Masriera (1901-1981) and the Role of Science Popularisation under Franco Regime. Science in Context. 2013. 26 (3): 527-549. 
Archiveros para Oficiales y Jefes del Ejército visitaron las instalaciones del SQUB, «modelo de perfección técnico-administrativa» ${ }^{29}$.

En 1975 las organizaciones químicas de Cataluña -Colegio de Químicos, Universidades Central y Autónoma de Barcelona, el IQS y otros organismosrindieron un merecido homenaje a María con motivo de su jubilación ${ }^{30}$. Dos años después, Josep Tarradellas, Presidente de la Generalitat, le entregaba la Medalla de Plata de la Universidad ${ }^{31}$. Finalmente, en marzo de 1980, con la presidencia del rector de la Universidad de Barcelona, Antoni Badía i Margarit (n. 1920), la Societat Catalana de Ciències Físiques, Químiques i Matemàtiques, le rendía un nuevo agasajo en la Facultad de Química ${ }^{32}$.

\section{Las publicaciones}

Entre 1943 y 1979 María Serrallach publicó 35 trabajos, de los cuales en uno no me consta impresión. Se trata de una conferencia impartida en 1969 en la Facultad de Farmacia de Barcelona. De los 34 restantes, 18 fueron folletos, trece artículos de revista, dos libros y un capítulo de libro. Al final de este punto comentaré una monografía, editada anónimamente en 1964, en la que María tuvo gran protagonismo, aunque no la he computado como publicación propia por carecer de firmantes. Cronológicamente, pues, María produjo hasta 1980 prácticamente un documento al año sobre documentación científica y química.

Pueden distinguirse varios campos de interés en sus publicaciones. El mayor número de sus aportaciones está consagrado al SQUB (24 contribuciones, una de ellas una conferencia que se imprimió). María se empeñó desde 1962 en dar a conocer la existencia del SQUB, en difundir los recursos documentales de que disponía el Seminario y también en publicitar la labor investigadora de las Facultades que lo integraban. Sobre el primer aspecto dio a luz siete textos e impartió una conferencia ${ }^{33}$, acerca del segundo editó

29. Actividades de la Biblioteca Universitaria y Provincial de Barcelona. Boletín de la Dirección General de Archivos y Bibliotecas. 1966; 15 (88): 20.

30. Homenaje a María Serrallach. La Vanguardia Española. 24 Dic 1975: 36.

31. Doña María Serrallach, una vida al servicio de la química. La Vanguardia Española. 26 Nov 1977: 20.

32. La Vanguardia Española. 13 marzo 1980: 28.

33. Consideraciones sobre la organización de una biblioteca especializada moderna. Biblioteconomía. 1950; 7 (25): 8-12; Serrallach, nota 2, artículo en el que presta gran atención a los sistemas 
cinco, en los que notificó los fondos de revistas y obras de referencia del SQUB en los años 1962 (da cuenta de 580 colecciones de revista), 1963, sobre obras de consulta en colaboración con su sobrina Enriqueta Serrallach, 1967, juntamente con Montserrat Brasó Pujol (con 243 títulos), 1972 (857 revistas) y 1979, ya mencionado, estos dos últimos en colaboración con Ana María Planet ${ }^{34}$. Sobre la última vertiente editó entre 1962 y 1973 doce folletos con una relación de las publicaciones realizadas por las cátedras de química de las Facultades de Farmacia y Ciencias de Barcelona ${ }^{35}$.

A su experiencia americana dedicó otros cuatro trabajos y una conferencia, que también se editó. Además del ya citado sobre el repertorio Chemical Abstracts, resultan muy interesantes sus apreciaciones sobre el Midwest Inter-Library Center (MILC), un organismo con edificio propio en Chicago creado en 1949 con el fin de racionalizar el gasto - evitando suscripciones y adquisiciones duplicadas que se consideraban innecesarias $-\mathrm{y}$ coordinar la labor de las 16 bibliotecas que formaban parte inicialmente del consorcio. El MILC, pues, actuaba como enlace de una red de bibliotecas académicas $^{36}$.

En este apartado hay que incluir también su análisis de las bibliotecas americanas y de sus profesionales, cuestión a la que dedicó dos trabajos

de automatización de los fondos de una biblioteca; La Biblioteca del Seminario de Química de la Universidad de Barcelona. Miscellanea Barcinonensia. 1966; n. 14; con el mismo título en: Boletín de la Dirección General de Archivos y Bibliotecas. 1968; 17 (119): 11-12, que es el que he consultado; Uno de los primeros servicios de documentación científica y técnica en España. In: Sarriá Rueda, Amalia, ed. Actas y Ponencias del II Congreso Nacional de Bibliotecas (Gerona, 3-8 de octubre de 1966). Madrid: Asociación Nacional de Archiveros, Bibliotecarios y Arqueólogos; 1968, p. 37-41; Serrallach, n. 6.

34. Revistas existentes en la Biblioteca del Seminario de Química de la Universidad de Barcelona, en marzo de 1962. Barcelona: Graphic Andros; 1962; Fondos de información y referencia que se reciben en la biblioteca del Seminario de Química de la Universidad de Barcelona. Barcelona: Graphic Andros; 1963; Revistas que se reciben en el Seminario de Química de la Universidad de Barcelona, en mayo de 1967. Barcelona: s.e.; 1967; Publicaciones periódicas existentes en la Biblioteca del Seminario de Química de la Universidad de Barcelona en junio de 1972. Barcelona: Seminario de Química; 1972; y Publicaciones periódicas existentes en la Biblioteca del Seminario de Química de la Universidad de Barcelona en marzo de 1979. Barcelona: Seminario de Química de la Universidad; 1979.

35. S.Q.U.B.: publicaciones de las Cátedras de Química de las Facultades de Ciencias y Farmacia de la Universidad de Barcelona: información bibliográfica. 12 vols., Barcelona: Seminario de Química; 1962-1973.

36. El M.I.L.C., una biblioteca para las bibliotecas. Biblioteconomía. 1957; 14 (45-46): 12-16. Sobre los primeros años de esta «biblioteca de bibliotecas»: Kaplan, Louis. The mid-west inter-Library Center, 1949-1964. Journal of Library History. 1975; 10 (4): 291-310. 
y una conferencia que dio en la Biblioteca Central de la Diputación de Barcelona para la delegación de la Asociación Nacional de Bibliotecarios, Archiveros y Arqueólogos en Cataluña y Baleares el 11 de mayo de $1958^{37}$ y que se editó en el Boletín de la Dirección General de Archivos y Bibliotecas ese mismo año ${ }^{38}$. En todos ellos recalca la funcionalidad de las bibliotecas estadounidenses, su eficacia, la atención que las bibliotecas técnicas prestan a la producción hemerográfica de los entonces países socialistas, la posibilidad de la suscripción de revista en microfilm, el recurso a fichas perforadas u otros procedimientos mecánicos para la selección de las adquisiciones, el papel preponderante que tiene el lector en la buena marcha de las bibliotecas, la planificación de las nuevas bibliotecas pensadas en el futuro lector, como suelos insonoros, luz cenital y difusa etc. Como señala María «las bibliotecas americanas no esperan a que el lector acuda».

En cuanto al personal, señala que todos los profesionales se han formado en alguna de las 35 escuelas de bibliotecarios que existen en el país y que, al término de sus estudios, algunos se especializan para poder trabajar en bibliotecas técnicas. En resumen

«Las bibliotecas americanas son... ricas en medios, en eficiencia del personal, ricas en contenidos antiguos y modernos, ricas en publicaciones de obras americanas de referencia para los lectores y ricas en programas de servicio y proyectos de realizaciones futuras» ${ }^{39}$.

Sobre bibliografía científica y química en general Serrallach editó tres trabajos. El primero es un artículo aparecido en 1943 en Afinidad, en el cual hace un exhaustivo análisis de los soportes bibliográficos que precisa cualquier profesional que quiera dedicarse a la investigación en química ${ }^{40}$.

La publicación que sin duda le reportó más prestigio dentro y fuera de España fue una extensa monografía sobre bibliografía química, editada en 1946, con un prólogo del catedrático de Química de la Universidad de Barcelona José Pascual Vila ${ }^{41}$. Es, sin duda, el primer tratado de documentación química publicado en nuestro país. La obra está estructurada en

37. Las Bibliotecas y los bibliotecarios en Norteamérica. Biblioteconomía. 1958; 15 (47): 5-22.

38. Las bibliotecas americanas y su personal. Boletín de la Dirección General de Archivos y Bibliotecas. 1958; 7 (46): 47-51.

39. Serrallach, n. 38, p. 51

40. Orientación bibliográfica básica en la investigación química. Afinidad. 1943; 20 (3-4): 60-73.

41. Bibliografía Química: documentación científico-industrial. Barcelona: Clarasó; 1946. 
catorce secciones en las que María analiza entre otros asuntos la literatura química, fuentes bibliográficas fundamentales en química orgánica e inorgánica, revistas de índices, revistas generales y especializadas, abreviaturas de títulos de revistas, abreviaturas y terminología inglesa y alemana, catálogo de revistas de Barcelona, índice de empresas y un muy detallado índice general alfabético. El libro fue reseñado en Estados Unidos en 1947 en el Journal of Chemical Education y era bien conocido en algunos círculos bibliotecarios de ese país ${ }^{42}$. Según María, la obtención de la beca americana fue facilitada, en parte, por la publicación de este tratado ${ }^{43}$. La Bibliografía fue reseñada también, en 1950, por A. Michael en la revista alemana Angewandte Chemie ${ }^{44}$.

Veinte años después, en colaboración con Lidia Benítez y Montserrat Brasó, ofreció un estudio acerca de las revistas científicas y técnicas españolas relacionadas con la química y disciplinas afines ${ }^{45}$.

En cuestiones teóricas y organizativas en documentación científica se centró en dos ocasiones. Un aspecto no excesivamente tratado por las bibliotecas científicas era el almacenaje y catalogación de folletos, recortes de prensa y otros soportes informativos poco habituales en bibliotecas científicas. Esta cuestión la trató María en un artículo publicado en $1963^{46}$. En 1969 impartió una charla a los alumnos de la asignatura Farmacia Galénica de la Facultad de Farmacia Universidad de Barcelona, en la que abordó cuestiones generales sobre información científica, y que ha quedado inédita ${ }^{47}$.

El último apartado lo conforman dos trabajos de temática diversa. En «Biblioteca de una industria farmacéutica», artículo aparecido en 1959 en

42. Journal of Chemical Education.1947; 24 (12): 620-621; Bledsoe, Elaine Austin (1957). Spanish Special Librarian Arrives under Jointly-Sponsored Program. Special Libraries. 1957; 48 (2): 74.

43. Serrallach, n. 15, f. 12.

44. Michael, A. Literatur. Bibliografía Química. Documentación científico-industrial, von María Serrallach. 1 Aufl. Barcelona, 1946, 130 Ptas. Angewandte Chemie. 1950; 62 (4): 103.

45. Serrallach, María; Benítez, Lidia; Brasó, Montserrat. Revistas científicas y técnicas españolas relacionadas con la química y afines. Barcelona: Seminario de Química de la Universidad de Barcelona; 1966.

46. Serrallach, María. Archivos verticales en bibliotecas técnicas y especializadas. Boletín de la Dirección General de Archivos y Bibliotecas. 1963; 13 (72-73): 29-32.

47. Serrallach, María. Información científica. Conferencia dada a los alumnos de Farmacia Galénica de la Facultad de Farmacia de la Universidad de Barcelona [1969] 17 f. No me ha sido posible la consulta de este texto, pues se halla excluido del préstamo interbibliotecario. Lo cito por la referencia en el Catàleg de Biblioteques de la Universidad de Barcelona [citado: 20 Mar 2012]. Referencia en: http://www.bib.ub.edu/es/. 
la revista La Industria Farmacéutica, Serrallach comienza por destacar el papel central que toda biblioteca tiene en un centro de investigación. Sólo una biblioteca bien organizada puede resolver los problemas informativos de quienes trabajan en un centro de ese tipo, químicos, microbiólogos, físicos y biólogos, entre otros. Con el fin de facilitar el trabajo de estos profesionales, Serrallach aborda el papel de las revistas, los repertorios de bibliografía corriente y el interés de las enciclopedias de química y los diccionarios lingüísticos, entre otros muchos aspectos. En el siguiente artículo, de 1972, ofrece un acercamiento general sobre las bibliotecas científicas y técnicas ${ }^{48}$.

En 1964 la Biblioteca Central de la Diputación de Barcelona le encargó presidir una comisión al objeto de inventariar las publicaciones periódicas que se recibían en las bibliotecas de la ciudad. El resultado fue la edición de una monografía, editada por dicha Biblioteca Central, en la que se recogen un total de 6.471 revistas ${ }^{49}$.

\section{La actividad docente}

Durante los cursos 1960-1961 y 1963-1964 me consta que María Serrallach impartió la docencia de la asignatura «Documentación Científica y Técnica» en la Escuela de Bibliotecarias de la Diputación de Barcelona, una importante novedad en el panorama español, pues por primera vez, y en una fecha tan temprana, la enseñanza de la Documentación Científica se ofrecía en un centro de formación bibliotecario por una experta en el tema. En el programa del curso 1960-1961, por ejemplo, se abordaban cuestiones como Obras de referencia científicas y técnicas, Centros y Organismos de Documentación e Investigación bibliográfica, Automatización, etc. ${ }^{50}$.

48. La Biblioteca de una industria farmacéutica. Industria Farmacéutica. 1959; 2 (9) (Consultado a través de su reseña en: Boletín de la Dirección General de Archivos y Bibliotecas. 1959; 8 (52): 105); Serrallach, María. Algunas impresiones sobre bibliotecas científicas y técnicas. Boletín de la Asociación Nacional de Archiveros, Bibliotecarios y Arqueólogos. 1972; 22, (1-4):111-116.

49. Biblioteca Central de la Diputación Provincial de Barcelona. Inventario de publicaciones periódicas que se reciben en las bibliotecas de Barcelona. Barcelona: Casa Provincial de Caridad-Imprenta Escuela; 1964. En esta monografía María se encargó, además, de inventariar los fondos de la Escuela de Ingenieros Industriales. Serrallach, n. 15, f. 11.

50. El Programa del curso 1960-1961 en: Rodríguez Parada, Concepción. L'ensenyament de la Biblioteconomia a Barcelona: de l'Escola a la Facultat de Biblioteconomia i Documentació. BiD, n. 9 [publicación seriada en Internet] 2002 [citado 22 junio 2012]. Disponible en: http:// 
Que María participara en las actividades académicas de la Escuela con la enseñanza de materias relacionadas con la documentación científica no era excepcional. Conviene recordar que la Escuela estuvo también abierta a que científicos complementaran la docencia que en la misma se impartía. Enric Freixa i Pedrals (1911-2002), ingeniero industrial formado en la Escuela de Barcelona, enseñó Historia de la Ciencia a partir del curso 1943-1944, en cuyo temario también trató de los sistemas de clasificación de los libros científicos y técnicos. Años después, desde mediados de los cincuenta, Freixa asumió además la enseñanza de Terminología Científica. Cuando dejó la Escuela continuó su labor al frente de esas asignaturas el también ingeniero industrial Lluis Marquet i Ferigle (1937-2011)

La enseñanza oficial y reglada de contenidos documentales en otros centros nacionales fue diferente y en algún caso más tardía que en Cataluña. El CSIC, por ejemplo, desde octubre de 1943 contaba con una Escuela de Auxiliares de la Investigación, llamada inicialmente «Estudios para Bibliotecarios Auxiliares», con el objetivo de formar un personal que conociera las técnicas básicas de laboratorio y los rudimentos de la bibliografía, humanística o científica. Hasta la promoción de 1945 no hubo enseñanzas científicas. La Escuela fue, en el fondo, un instrumento para formar personal administrativo auxiliar del cual el CSIC estaba muy necesitado ${ }^{52}$.

Una orden ministerial de 23 de septiembre de 1964 creaba la Escuela de Documentalistas, vinculada a la Dirección General de Archivos y Bibliotecas y con sede en la Biblioteca Nacional, que estuvo operativa hasta 1986. El objetivo de la Escuela era «...formar a los futuros profesionales de la Documentación en sus diversos grados, tanto con vistas a su posible incorporación a las empresas particulares, como para opositar a las plazas de la Administración ${ }^{53}$, es decir, se pretendía fomentar un profesional más

www.ub.es/biblio/bid/09rodri1.htm. De su participación en el año académico 1963-1964 hay una breve noticia en: La Srta. Serrallach profesa la asignatura de Documentación Científica y Técnica. Boletín de la Dirección General de Archivos y Bibliotecas 1963; 12 (72-73): 58.

51. Puig Rovira, Francesc X.; Puig-Pla, Carles. Enric Freixa i Pedrals (1911-2002). Barcelona: Col.legi d'Enginyers Industrials de Catalunya; 2012, p. 33-34. Esta actitud positiva de la Escuela hacia la ciencia se aprecia también en Biblioteconomía, su revista, en la que desde mediados de los cuarenta se hicieron frecuentes los artículos y noticias sobre documentación científica, tanto de bibliotecarias como de científicos, como del ya mencionado Enric Freixa.

52. Tortajada, Amadeo. Escuela de Estudios Auxiliares de la Investigación. Ayer, hoy y su mañana. Arbor. 1974: 89 (345-346): 121-131.

53. Ante el próximo curso 1964-65 de la Escuela de Documentalistas. Boletín de la Dirección General de Archivos y Bibliotecas. 1964; 13 (78): 7-10. 
en la línea de los special librarians anglosajones. En el Plan de Estudios de Ayudantes de Documentación se incluyó la disciplina «Fundamentos de Documentación» (3 horas semanales) impartida por Marcelina Iñiguez Galíndez (Directora de la Biblioteca del Ministerio de Información y Turismo), y en el Plan de Estudios de Documentalista, «Documentación», a cargo de Javier Lasso de la Vega, Exdirector de la Biblioteca General de la Universidad Central, con una carga lectiva también de tres horas semanales ${ }^{54}$. Lasso de la Vega expuso el programa de su asignatura en un artículo de revista, apoyado bibliográficamente, entre otros autores, en Erich Pietsch (1902-1979) - uno de los creadores de la documentación científica en la Europa de la posguerra-, con un total de cuarenta temas en los que estudiaba pormenorizadamente las cuestiones fundamentales referidas a la disciplina, con algunas lecciones dedicadas a la documentación científica, tanto en sus vertientes teórica como organizativa ${ }^{55}$.

La fundación de esta Escuela y el Congreso Nacional de Bibliotecas, celebrado en Gerona en octubre de 1966, son dos buenos indicadores del paulatino cambio que estaba experimentando el muy tradicional Cuerpo de Bibliotecarios, Archiveros y Arqueólogos. En dicha reunión la Primera Ponencia trató de las «Bibliotecas y la Documentación en el desarrollo científico y técnico» y fue desarrollada por Javier Lasso de la Vega. A la misma se presentaron cuatro comunicaciones, de María Serrallach, «Uno de los primeros servicios de Documentación Científica y Técnica de España», sobre el SQUB, que ya he comentado; una segunda a cargo de Montserrat Brasó, también de Barcelona, que abordó el tema «La Biblioteca de una Asociación profesional y su documentación», la tercera acerca de «La Documentación en las bibliotecas empresariales» y que fue expuesta por María del Carmen Mayol y María Forn Homar, también de Barcelona, y, finalmente un trabajo de José Antonio de Artigas, de Madrid en torno a la cuestión «Documentadores y Documentalistas» ${ }^{56}$. El nombramiento de Luis Sánchez Belda en 1968 como Director General de Archivos y Bibliotecas fue un auténtico revulsivo, que se manifestó, entre otros aspectos, en un

54. La Escuela de Documentalistas ante el Nuevo curso 1965-1966. Boletín de la Dirección General de Archivos y Bibliotecas. 1965; 14 (84-85): 30.

55. Lasso de la Vega, Javier. Documentación. Programa de esta asignatura en la Escuela de Documentalistas. Boletín de la Dirección General de Archivos y Bibliotecas. 1966; 15 (90-91): 32-37.

56. El II Congreso Nacional de Bibliotecas. Gerona 3 a 8 de octubre de 1966. Boletín de la Dirección General de Archivos y Bibliotecas. 1968; 15 (92): 2-14. 
cambio sustancial de la orientación de la revista del Cuerpo hasta 1974, fecha en que dejó la Dirección General. El número de trabajos de temática documental y científica editados en la revista creció de una manera espectacular durante su mandato ${ }^{57}$.

\section{El Seminario de Química (1937-1984)}

En abril de 1936 José Deulofeu Poch (1879-1957), catedrático de Química Inorgánica en la Universidad de Barcelona, y otros profesores de las Facultades de Farmacia y Química, promovían la creación de la Biblioteca Especializada de Química Antoni Martí, resultado de la integración de los fondos bibliográficos de las Facultades de Química y Farmacia ${ }^{58}$. Un año después esta biblioteca pasaba a denominarse Seminario de Química de la Universidad de Barcelona. En su creación pesaron básicamente a dos motivos. El primero, la proximidad física y temática de ambas bibliotecas. Por otro, la necesidad de racionalizar los gastos, con la cual se evitaban duplicaciones innecesarias.

Durante más de 30 años el SQUB estuvo ubicado en un espacio situado en el patio de la Facultad de Ciencias, en el edificio central de la Universidad, hasta que en 1970 se realojó en un amplio local de casi dos mil metros cuadrados de superficie, con capacidad para albergar más de cincuenta mil volúmenes, en el nuevo campus de Pedralbes ${ }^{59}$.

Aunque el SQUB nació, tal como ya he señalado, como soporte para la labor investigadora de los docentes de las dos Facultades que promovieron su fundación, quiso ser también una biblioteca abierta a los estudiantes y a cualquier usuario que precisara consultar sus fondos, especialmente los que trabajaban en las empresas químicas ${ }^{60}$. Esta vocación de servicio es la

57. Olagüe de Ros, Guillermo. Luis Sánchez Belda (1920-1984) y su contribución al desarrollo de la documentación científica en la España contemporánea. In: García Caro, Concepción; Vilchez Pardo, Josefina, coords. Homenaje a Isabel de Torres Ramírez. Estudios de Documentación dedicados a su memoria. Granada: Editorial de la Universidad; 2009, p. 557-571.

58. Sales, Joaquim. La Química a la Universitat de Barcelona. Barcelona: Universitat; 2011, p. 141. Los otros promotores fueron Salvador Brosa, José Amargós, José Pascual, Miguel Masriera, Ramón Casamada y Cándido Torres (Alcolea, Lamarca, Llopart, et al., n. 19, p. 69).

59. Notas Universitarias. Inauguración de la Biblioteca del Seminario de Química. La Vanguardia Española. 23 diciembre 1970: 35; Alcolea, Lamarca, Llopart et al., n. 19, p. 71

60. Alcolea, Lamarca, Llopart et al., n. 19, p. 69-70. 
que posibilitó que el SQUB consiguiera financiación del mundo empresarial catalán. En 1948, y tras vencer resistencias de algunos profesores, 33 industrias aportaron 10.600 pesetas. En 1967 libraron ya 185.000 pesetas (más del 25\% del presupuesto total de ese año, que ascendía a 700.000 pesetas). En 1972 el número de entidades colaboradoras había ascendido a 133, con un montante de 1.028 .000 pesetas ${ }^{61}$. También el número de lectores creció de manera significativa. En el sexenio 1966-1973 contó con 65.065 usuarios, duplicándose casi su número entre el primero y último año (7.132 y 13.021, respectivamente) ${ }^{62}$. El soporte económico de la Universidad y de la industria hizo posible que la biblioteca dispusiera de la única colección completa del Chemical Abstracts de todo el Estado español, al igual que del repertorio de química orgánica de Beilstein.

En 1984 se disolvía el SQUB. A partir de entonces, las Facultades de Física y Química, ubicadas en el mismo edificio, aunaron sus esfuerzos documentales y crearon la Biblioteca de Física y Química de la Universidad de Barcelona (BFQUB), que albergaría los fondos de Física, Química, Ingeniería de Materiales, Ingeniería Electrónica e Ingeniería Química. La nueva biblioteca se repartía en varias salas: una para estudiantes, con libros de texto; dos salas de revistas, la de Física con 190 revistas vivas y la de Químicas con 250, además de otras 1.200 colecciones, y salas de trabajo y servicios. La nueva biblioteca ofrecía diversas prestaciones: fotocopias, obtención de documentos originales (nacionales o extranjeros), traducciones, patentes y consulta teledocumental, con dos ordenadores conectados a once suministradores de bases de datos (fundamentalmente, CAS on line, ESA, DIALOG y DIMDI, entre otros $)^{63}$.

Durante un largo periodo de la historia del SQUB María Serrallach fue la única responsable. En las memorias de la Universidad no aparece otro personal hasta 1974, en la que figuran, además de María, Ana María

61. Serrallach, 1968, n. 33. Serrallach, n. 15, f. 8. En 1966 el presupuesto del IQS era muy parecido al del SQUB (600.000 pesetas). Asamblea General de Patronos en el Instituto Químico de Sarriá. ABC. 1966; 24 de febrero, p. 67-68.

62. Universidad de Barcelona. Memorias correspondientes a los cursos académicos 1965-1966, 1966-1967, 1967-1968, 1968-1969, 1971-1972 y 1972-193 [consultadas 14 mayo 2012]. Disponibles en: Dipòsit Digital de la UB. Anuaris i Mèmories. http://diposit.ub.edu/dspace/ handle $/ 2445 / 961 /$ browse?type=dateissued\&submit_browse=Fecha+Publicaci\%C3\%B3n .

63. En 1988 el número de consultas teledocumentales realizadas por la BFQUB ascendió a 488, de las cuales el 50\% fueron a petición de la industria, un 25\% por la Universidad y el resto por particulares. Planet Rabascall, Anna María. La Biblioteca de Física y Química de la Universidad de Barcelona, abierta a la industria. Política Científica. 1988; n. 15: 55-56. 
Planet, como bibliotecaria, Enriqueta Serrallach Milá, química documentalista, y una auxiliar, Núria Codorniu Rovelló. Diez años después, y con el fin de cubrir las necesidades de personal, la Biblioteca amplió su plantilla a nueve bibliotecarias (una de ellas como coordinadora, Anna María Planet Rabascall, antigua colaboradora del SQUB). Una vez se constituyó la BFQUB la química documentalista asumió la responsabilidad de las búsquedas teledocumentales ${ }^{64}$.

\section{Colofón}

En Cataluña ha existido una larga tradición de coordinación de servicios documentales en las diferentes ramas de la ciencia, bien con carácter global, con la idea de crear un centro catalán, como el promovido por la Associació d'Enginyers Industrials de Catalunya en $1978^{65}$, o bien en áreas concretas, como la Coordinadora de Documentació Biomèdica, en cuya puesta en marcha en 1983 jugó un determinante papel Domenèc Turuguet i Mayol (1924-2003) ${ }^{66}$. Cuando se instalaron en España las primeras terminales de teledocumentación, Cataluña contó tempranamente con una operativa en el Consorci de Documentació de Catalunya desde enero de 1974, un organismo creado en 1969 y que merecería un estudio más profundo.

64. Planet, Anna Ma Biblioteca de Física i Química de la Universitat de Barcelona. Butlletí de I'Associació de Bibliotecaris de Catalunya. 1985; n. 7: 65-67.

65. Abella i Espar, Ma Teresa; Piñol i Espí, Pepita. La Informació i Documentació Científica i Tècnica a Catalunya. Alternativa per la creació d'un SISTEMA NACIONAL D'INFORMACIÓ [1978] 158 f. [citado: 20 marzo 2012]. Disponible en http://www.bib.ub.edu/es/. Según datos de este estudio existían por entonces en Cataluña una veintena de tales centros, escasamente relacionados entre sí, al igual que los del resto del Estado español.

66. Tengo intención en un futuro inmediato de aproximarme a la significación histórica en el ámbito de la documentación científica de Domènec Turuguet i Mayol, un especialista en medicina del trabajo que realizó importantes aportaciones al estudio de las enfermedades profesionales en nuestro país. Señalaré que desde 1972 Turuguet dirigió el Centro Nacional de Información y Documentación, adscrito al Instituto de Higiene y Seguridad en el Trabajo de Barcelona. En dicho Centro Turuguet impartió cursos sobre documentación científica, en colaboración con las bibliotecarias del mismo. Una breve nota biográfica en: Castejón, Jordi. Domènec Turuguet i Mayol (1924-2003). In Memoriam. Archivos de Prevención de Riesgos Laborales. 2004; 7 (1), 28-29. Una nota sobre el Centro en: Turuguet y Mayol, Doménec. Consideracions respecte al traspas del Centre Nacional de Informació i Documentació des del Servei Social d'Higiene i Seguretat del Treball a la Generalitat. In: Primeres Jornades de Sanitat del PSUC. Ponencies i Comunicats. Barcelona: Comissió de Sanitat del Comitè Central del PSUC; 1978, p. 152-153. 
El SQUB fue el primer intento de coordinación bibliotecaria en la Universidad de Barcelona. Cuando se disolvió en 1984, se mantuvo al integrarse las bibliotecas de las Facultades de Físicas y Químicas para constituir la BFQUB. Un paso más se dio en 2004 al constituirse el CRAI, el Centre de Recursos per a l'Aprenentatge i la Investigació, que totalizó 18 bibliotecas de la Universidad de Barcelona, y que nació como respuesta a la puesta en marcha del Espacio Europeo de Educación Superior.

María Serrallach fue una adelantada a su tiempo, una bibliotecaria con clara vocación documentalista, que muy tempranamente convirtió el SQUB en un modélico servicio de documentación química, buscó el apoyo económico de la industria catalana para su sostenimiento y mantuvo estrechas relaciones profesionales con otros organismos, catalanes y de ámbito nacional.

\section{Agradecimientos}

A Isabel Parés y Anna María Planet Rabascall, por su valiosa información acerca de María Serrallach. Y a Jordi Castejón Castejón, por su aportación sobre Domènec Turuguet i Mayol. 